J. Clin. Chem. Clin. Biochem.

Vol. 20, 1982, pp. 907-914

\title{
Interferon: \\ Wesentlich vereinfachte, enzym-immunologische Bestimmung mit zwei monoklonalen Antikörpern
}

\author{
Von $H$. Gallati \\ Zentrale Forschungseinheiten F. Hoffmann-La Roche \& Co., Ltd., Basel, Schweiz
}

(Eingegangen am 28. April/5. Juli 1982)

Herrn Prof. Dr. Dr. Walter Boguth zum 65. Geburtstag

Zusammenfassung: Basierend auf dem Festphasen Sandwich-Prinzip erfolgt die enzym-immunologische Bestimmung des humanen Leukocyten-Interferons (HuIFN- $\alpha$ ) mit einer wesentlich vereinfachten Testmethode. Dabei wird das Interferon der Proben und der mitzuführenden Standardlösungen während eines einzigen Inkubationsschritts einerseits an eine mit monoklonalem (Maus) Anti-Interferon beschichtete-Polystyrolkugel gebunden und andererseits mit einem zweiten monoklonalen (Maus) Interferon-Antikörper, an den Meerretich-Peroxidase gekoppelt ist, markiert. Nach dieser immunologischen Inkubation wird das nicht gebundene Material durch Waschen entfernt und anschließend die Menge der an die Kugel gebundenen Peroxidase enzymatisch gemessen. Die dabei entstandene Farbintensität wird photometrisch bestimmt und ist der Interferon-Konzentration in den Proben und den Standardlösungen direkt proportional.

Die Nachweisgrenze dieses enzym-immunologischen Tests kañn je nach Art der Testdurchführung bis 100 I.U./1 Interferron gesteigert werden. Der Variationskoeffizient in der Serie beträgt 3-6\%. Die Serumproben können ohne jede Vorbehandlung direkt für die Interferon-Bestimmung eingesetzt werden. Die Inkubationsdauer beträgt je nach gewünschter Testempfindlichkeit zwischen 30 Minuten und 24 Stunden.

\section{Interferon: A greatly simplified, enzyme-immunological determination, using two monoclonal antibodies}

Summary: Human leukocyte interferon (HuIFN- $\alpha$ ) can be determined by a substantially simplified enzyme-immunological method, based on the solid phase sandwich principle. In a single step, the interferon in the sample or the standard solution (tests and standards are run concomitantly) is bound to a polystyrene bead coated with monoclonal (mouse) anti-interferon, and at the same time it is labelled with a second monoclonal (mouse) interferon antibody, which is coupled to horse radish peroxidase. After this immunological incubation, the non-bound material is removed by washing, and the quantity of peroxidase bound to the bead is measured enzymically. The resulting colour intensity is determined photometrically, and it is directly proportional to the interferon concentration in the sample.

The detection limit of the test can be as low as 100 İ.U./l interferon, depending on the assay conditions. The variation coefficient within series was 3-6\%. Serum samples can be used directly in the test without pretreatment. Depending on the required sensitivity, the incubătion may be performed for periods between 30 minutes and 24 hours.

\section{Einfuihrung}

Dàs Interferon wurde 1957 von Isaacs \& Lindemann als eine natürliche Abwehrsubstanz gegen virale Infektionen beschrieben (1).

Heute kennt man eine Vielzahl von Interferonen, die als niedermolekulare Glycoproteine charakterisiert werden und eine außerordentlich hohe biologische Aktivität besitzen. Durch den Kontakt mit Viren, mit deren
Nucleinsäuren oder auch mit anderen Substanzen können verschiedene Zellen im Organismus der Wirbeltiere dazu stimuliert werden, Interferone zu bilden und zu sezernieren. Als interzelluläre Wirksubstanzen erhöhen die Interferone die Virusresistenz der Zellen, hemmen das Wachstum und die Teilung normaler und maligner Zellen, aktivieren die natürlich vorkommenden Killerzellen und stimulieren spezielle Immunsysteme. (Ubersichtsartikel: 1.c. (2-9)). 
Die Leukocyten synthetisieren nach viraler Induktion über 10 verschiedene Interferone. Diese Leukocyteninterferone (HuIFN- $\alpha$ ) können heute mit Hilfe der Gentechnologie in größeren Mengen hergestellt werden (10-13). In der vorliegenden Arbeit wird eine enzymimmunologische Bestimmungsmethode für in Bakterien (E. coli) durch DNA-Rekombinationstechnik hergestelltes Leukocyteninterferon (HuIFN- $\alpha_{\mathrm{r}}$ ) beschrieben.

Das bisherige Nachweisverfahren (14-15) basiert auf der antiviralen oder der cytopathischen Aktivität des Interferons. Die Resultate werden in internationalen Einheiten (I.U.) angegeben. Eine I.U. ist definiert als der Kehrwert jener Interferon-Verdünnung, die entweder die Virusplaquebildung zu 50\% hemmt oder $50 \%$ des Zellrasens schützt. Eine I.U. entspricht etwa 10 Attomol Leukocyteninterferon (9). Der biologische Interferontest ist arbeitsaufwendig und komplex in der Durchführung. Er ist auch störanfällig, indem neben Interferon noch andere Bestandteile in den Proben das Zellen- oder das Viruswachstum beeinflussen können.

$$
\text { i! }
$$

Nachdem monoklonale Interferon-Antikörper für die affinitäts-chromatographische Reinigung des Leukocyteninterferons hergestellt wurden (16), waren auch die Voraussetzungen für die Entwicklung eines radio-immunologischen (17) und eines enzym-immunologischen Interferontests gegeben (18).

Der hier beschriebene, enzym-immunologische Interferontest basiert auf dem Festphasen-Sandwich-Prinzip und wird mit einem wesentlich vereinfachten Testverfahren durchgeführt. Das Interferon der Proben sowie der mitzuführenden Standardlösungen wird während eines einzigen Inkubationsschritts einerseits an die mit einem monoklonalen (Maus) Interferon-Antikörper beschichtete Polystryrolkugel $(\phi=6.35 \mathrm{~mm})$ gebunden und andererseits mit einem zweiten monoklonalen (Maus) Interferon-Antikörper, an den Meerrettich-Peroxidase gekoppelt ist, markiert. Nach dieser immunologischen Reaktion wird das nicht gebundene Material durch einen Waschschritt entfernt und anschließend die Menge der an die Kugel gebundenen Peroxidase enzymatisch gemessen. Die dabei entstehende Farbintensität wird photometrisch bestimmt. Sie ist der Interferon-Konzentration in den Proben und den Standardlösungen direkt proportional.

Das Prinzip dieses wesentlich vereinfachten, immunologischen Testverfahrens isst in der Abbildung 1 schematisch dargestellt.

Zur enzym-immunologischen Interferon-Bestimmung werden in Teströhrchen $0,2 \mathrm{ml}$ Probe oder $0,2 \mathrm{ml}$ Interferon-Standardlösungen mit $0,05 \mathrm{ml}$ Anti:InterferonPeroxidase-Konjugatlösung gemischt, eine mit AntiInterferon sensibilisierte Polystyrolkugel zuggegeben und bei $18-26^{\circ} \mathrm{C}$ während 30 Minuten bis 24 Stunden inkubiert. Nach einem einfachen Waschschritt wird anschließend die immunologisch an die Kugel gebundene Peroxidase enzymatisch bestimmt und mit Hilfe der mitgeführten Standards der Interferongehalt in den Proben berechnet. Für die Interferon-Bestimmung brauchen die Proben nicht vorbehandelt zu werden. Die Nachweisgrenze dieser Testmethode kann maximál bis 100 I.U./I Înterferon gesteigert werden.

Zur Überwachung der Produktion und der Reinigung des Interferons kann die Dạuer der immunölogischen Reaktion auf 30 Minuten reduziert werden. Mit diesem „Rapid“-Test wird ein Meßbereich von $0-10^{6}$ I.U./1 bei einer Nachweisgrenze von $20 \times 10^{3}$ I.U. II İterferon erreicht.

Die vorliegende Arbeit berichtet über die Optimierung der enzym-immunologischen Interferon-Bestimmung.

Testprinzip (schematisch)

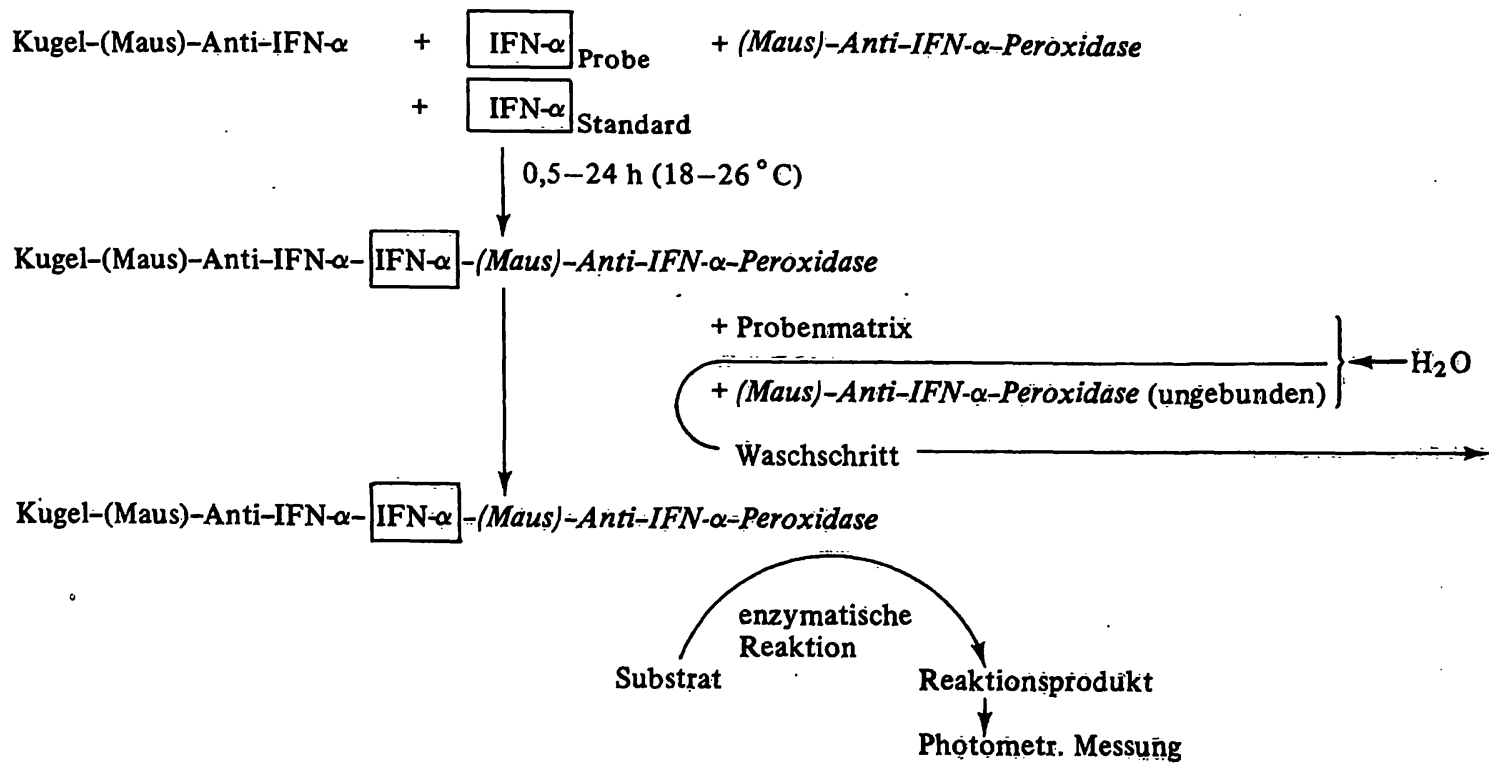

Abb. 1. Schematische Darstellung der enżym-immunologischen Interferonbestimmung naçh der „Eintopf"-Testmẹthode. 


\section{Material und Methoden}

\section{Reagenzien}

Die beiden monoklonalen (Maus) HuIFN- $\alpha_{\mathrm{r}}$-Antikörper (LI-1 und LI-9) wurden von Prof. Th. Staehelin, F. Hoffmann-La

Roche \& Co. Ltd., Basel, hergestellt. Die Antikörper wurden aus Ascitesflüssigkeit durch Ammoniumsulfat-Fällung und DEAE-Cellulose-Chromatographie isoliert.

Meerrettich-Peroxidase, ein lyophilisiertes Produkt vom Reinheitsgrad I, wurde von der Firma Boehringer, Mannheim, bezogen.

Die Polystyrolkugeln mit einem Durchmesser von $6,35 \mathrm{~mm}$ wurden von der Firma Spherotech AG, Zürich, und die Kunststoff-Teströhrchen $(11 \times 75 \mathrm{~mm})$ von der Firma Sarstedt geliefert.

Alle verwendeten Chemikalien waren von analytischer Reinheit.

\section{Proben und Standards}

Neben den Proben aus der bakteriell durchgeführten Produktion von Interferon (HuIFN- $\alpha_{\mathrm{T}}$ ) (Hoffmann-La Roche, Basel, Schweiz) wurden auch Humanseren von Blutspendern mit diesem Leukocyteninterferon (HuIFN- $-\alpha_{\mathrm{r}}$ ) aufgestockt und analysiert.

Als Interferon-Standards dienten einerseits eine Probe mit bakteriell gewonnenem Interferon und andererseits eine Lösung mit aus Blut gereinigtem Leukocy teninterferon. Beide Standards wurden vorgängig im biologischen, antiviralen Test bestimmt.

\section{Beschichtung von Polystyrolkugeln mit monoklonalem (Maus) Anti-HuIFN- $\alpha_{\mathrm{T}}$}

Basierend auf der Beschichtungsmethode von Voller et al. (19) wurden die Polystyrolkugeln batchweise in $50 \mathrm{mmol} / 1$ Natriumhydrogencarbonatlösung vom $\mathrm{pH} 8,2 \mathrm{mit} 0,1 \mathrm{~g} / 1$ monoklonalem (Maus) Anti-HuIFN- $\alpha_{\mathrm{T}}$ (LI-9) während 16 Stunden bei $18-26^{\circ} \mathrm{C}$ inkubiert. Anschließend wurden die sensibilisierten Kugeln mit destilliertem Wasser gewaschen und in $0,1 \mathrm{~mol} / 1 \mathrm{TRIS} / \mathrm{HCl}, \mathrm{pH}$ 7,5 bei $2-8^{\circ} \mathrm{C}$ aufbewahrt.

\section{Herstellung von Peroxidase-Konjugat mit monoklonalem (Maus) Anti-HuIFN- $\alpha_{\mathbf{r}}$}

Entsprechend der Nakane-Methode (20-22) wurden Zuckerreste der Peroxidase mit Natriummè taperiodat zu Aldehydgruppen oxidiert, die dann anschließend mit freien Aminogruppen des HuIFN- $\alpha_{r}$-Antikörpers reagierten. Mit Hilfe einer Sephacryl S-300 Chromatographie wurde das Anti-Interferon-PèroxidaseKonjugat von den freien Reaktionspartnern abgetrennt.

\section{Allgemeine Testvorschrift zur Durchfü̈hrung des enzym- immunologischen Interferontests}

In Sarstedt Teströhrchen $(11 \times 75 \mathrm{~mm})$ werden gegeben:

1. 0,2 ml Probe, unverdünnt bzw. mit dem ,Verdünnungspuffer" $(0,1 \mathrm{~mol} / 1$ Natriumphosphatpuffer vom $\mathrm{pH} 6,5 \mathrm{mit}$ $10 \mathrm{~g} / 1$ Rinderserumalbümin und $200 \mathrm{mi} / 1$ Humanserum) entsprechend verdünnt, oder

$0,2 \mathrm{ml}$ Interferon-Standardlösungen (0 I.U./1, 12,5 $\times 10^{3}$ I.U. $/ 1,25 \times 10^{3}$ I.U. $/ 1$ und $50 \times 10^{3}$ L.U. / I Interferon gelöst im ,Verdünnungspuffer").

2. 0,05 ml Testlösung (0,5 mg/1 Peróxidase-Konjugat mit monoklonalem (Maus) Anti-HuIFN- $\alpha_{r}$ in Humanserum mit $0,1 \mathrm{~mol} / 1$ Natriumphosphatpuffer vom $\mathrm{pH} 6,5$ ).

3. Polystyrolkugel, beschichtet mit monoklonalem (Maus) Anti-HulFN- $\alpha_{\mathbf{r}}$.

Nach einer Inkubation von $10-24$ Stunden bei $18-26^{\circ} \mathrm{C}$ werden die Kugeln dreimal mit destilliertem Wasser gewaschen, um das nicht gebundene Material zu entfernen. Die an die Kugel immunologisch gebundene Peroxidase wird anschließend bestimmt, indem zur gewaschenen Kugel im Teströhrchen $0,5 \mathrm{ml}$
Substratpuffer $(0,1 \mathrm{~mol} / 1$ Kaliumcitratpuffer vom pH 5,0 mit $6 \mathrm{mmol} / 1 \mathrm{H}_{2} \mathrm{O}_{2}$ und $40 \mathrm{mmol} / 1$ 1,2-Phenylendiamin) zugegeben und während genau 30 Minuten bei $18-20^{\circ} \mathrm{C}$ inkubiert wird. Die enzymatische Reaktion wird dann durch Zusatz von $0,5 \mathrm{ml}$ einer $4 \mathrm{~mol} / 1$ Salzsäure abgestoppt und die Farbintensität bei der Wellenlänge $492 \mathrm{~nm}$ innerhalb von 60 Minuten photometrisch bestimmt. Der Interferon-Gehalt der Proben wird an Hand der mitgefuihrten Standardlösungen berechnet.

\section{Resultate \\ Einfluß der Pufferart, der Pufferkonzentration, der Ionenstärke und des $p H$-Wertes auf den Interferon-EIA}

Unter sonst identischen Reaktionsbedingungen wurde der Einfluß der folgenden Puffersysteme bei einer jeweiligen Konzentration von $0,1 \mathrm{~mol} / 1$ untersucht: Triethanolamin, $\mathrm{pH} 7,5$; TRIS/HCl, $\mathrm{pH} 7,5$; Natriumborat, $\mathrm{pH}$ 7,0; Natriumphosphat, $\mathrm{pH} 6,5$; Natriumcitrat, $\mathrm{pH} 5,0$ und Natriumacetat, pH 5,0.

Mit Natriumphosphat werden bezüglich Testempfindlichkeit und Reagenzienleerwert die besten Resultate erzielt. Mit Natriumcitrat und Natriumacetat resultiert wegen dès tieferen $\mathrm{pH}$-Wertes ein hoher Reagenzienleerwert.

Innerhalb des untersuchten Konzentrationsbereichs von 25-400 mmol/1 hạt die Natriumphosphatkonzentration im pH-Bereich von 6,5 keinen signifikanten Einfluß weder auf die immunologische Reaktionskinetik, noch auf die Testempfindlichkeit, noch auf den Reagenzienleerwert. Die weiteren Versuche wurden daher mit einer Natriumphosphatkonzentration von $0,1 \mathrm{~mol} / 1$ durchgeführt.

Ein Zusatz̄ von Natriumchlorid zur Testlösung im Konzentrationsbereich von $0-400 \mathrm{mmol} / \mathrm{l}$ zeigt keinen Einfluß auf die Testresultate.

In weiteren Versuchen wurde die Abhängigkeit der immunologischen Reaktion vom pH-Wert der phosphatgepufferten Testlösung abgeklärt. Die Resultate, die in der Abbildung 2 zusammengestellt sind, zeigen ein starkes Absinken des Reagenzienleerwertes mit steigendem $\mathrm{pH}$-Wert, während die Testempfindlichkeit beim pH 6,5 am höchsten ist. Bei allen untersuchten $\mathrm{pH}$-Werten ( $\mathrm{pH} 4,5$ bis 8,5) konnte eine lineare Proportionalität der Farbintensität zur eingesetzten Interferon-Konzentration festgestellt werden. Für die weiteren Versuche wurde die Testlösung auf den $\mathrm{pH}$ 6,5 eingestellt.

\section{Humanserum als Matrix der Interferon-EIA-Testlösung}

Zur Optimierung der Reaktionsbedingungen wurden der Interferon-Testlösung unterschiedliche Mengen von Rinderserumalbumin (0-50 g/1), Rinderserumglobulin $(0-10 \mathrm{~g} / \mathrm{l})$, Rinderserum $(0-400 \mathrm{ml} / \mathrm{l})$, Ziegenserum $(0-400 \mathrm{ml} / \mathrm{l})$, Pferdeserum $(0-200 \mathrm{ml} / \mathrm{l})$ und Humanserum (0-1000 ml/1) zugesetzt. Zudem wurden diverse Mischungsverhältnisse der erwähnten Seren untersucht. 


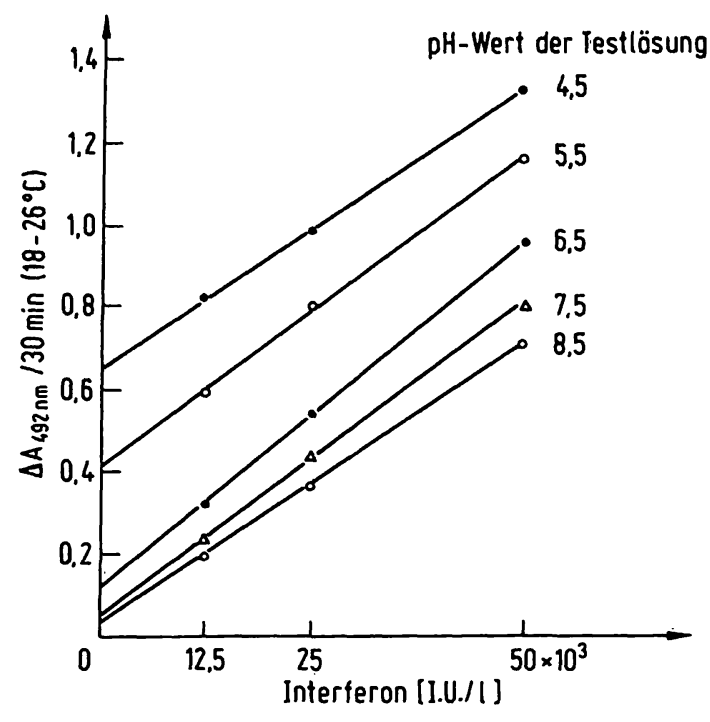

Abb. 2. Abhängigkeit des Interferon-EIA vom pH-Wert der Testlösung.

Der Interferon-EIA wurde nach der allgemeinen Testvorschrift durchgefuiht, außer daß der pH-Wert der AntiInterferon-Pẹroxidaselösung entsprechend variiert wurde.

Mit 100\% Humanserum als Matrix der Interferon-Testlösung werden die günstigsten Resultate bezüglich der Testempfindlichkeit, des Reagenzienleerwerts, der Reproduzierbarkeit, der Stabilisierung der enzymatischen und immunologischen Aktivität des Anti-HuIFN- $\alpha_{\mathrm{r}}$-PeroxidaseKonjugats wie auch der Praktikabilität der Testdurchführung erzielt.

Ein wesentlicher Vorteil der Verwendung von Humanserum als Matrix für die Testlösung besteht darin, daß der Interferon-EIA als "One-tube-Test" durchgeführt werden kann: Für die immunologische wie auch für die enzymatische Reaktion kann das gleiche T'eströhrchen benützt werden. Auf diese Weise muß nur ein einziges Röhrchen für jede Probe angeschrieben werden, und zudem entfällt das Transferieren der gewaschenen Kugel in ein frisches Teströhrchen.

Das für die Testlösung vorgesehene Humanserum wird vorgängig geprüft auf Interferon oder auf andere Substanzen, die den Interferon-EIA beeinflussen könnten.

\section{Abhängigkeit des Interferon-EIA vom Gehalt an Anti- HuIFN- $\alpha_{\mathrm{r}}$-Peroxidase-Konjugat .}

Für den Einsatz im Interferon-EIA muß das AntiHuIFN- $\alpha_{\mathrm{r}}$-Peroxidase-Konjugat eine möglichst hohe spezifische Immunaktivität wie auch Enzymaktivität besitzen.

Mit steigender Konzentration an Anti-HuIFN- $\alpha_{\mathrm{r}}$ Peroxidase-Konjugat in der Testlösung nimmt die Testempfindlichkeit zu, ohne daß der entsprechende Reagenzienleerwert wesentlich ansteigt (Abb. 3). mit Hilfe der Konjugatkonzentration kann somit die gewünschte Testempfindlichkeit wie auch der Interferon-Meßbereich eingestellt werden. Da bisher Anhaltspunkte über die Interferon-Konzentration im Blut fehlen, wurde der generelle Interferôn-EIA vorläufíg für einen Meßbereich von 0-50 X $10^{3}$ I.U./1 Interferon mit einer Nachweisgrenzé von etwa $1 \times 10^{3}$ I.U./1 ausgelegt.

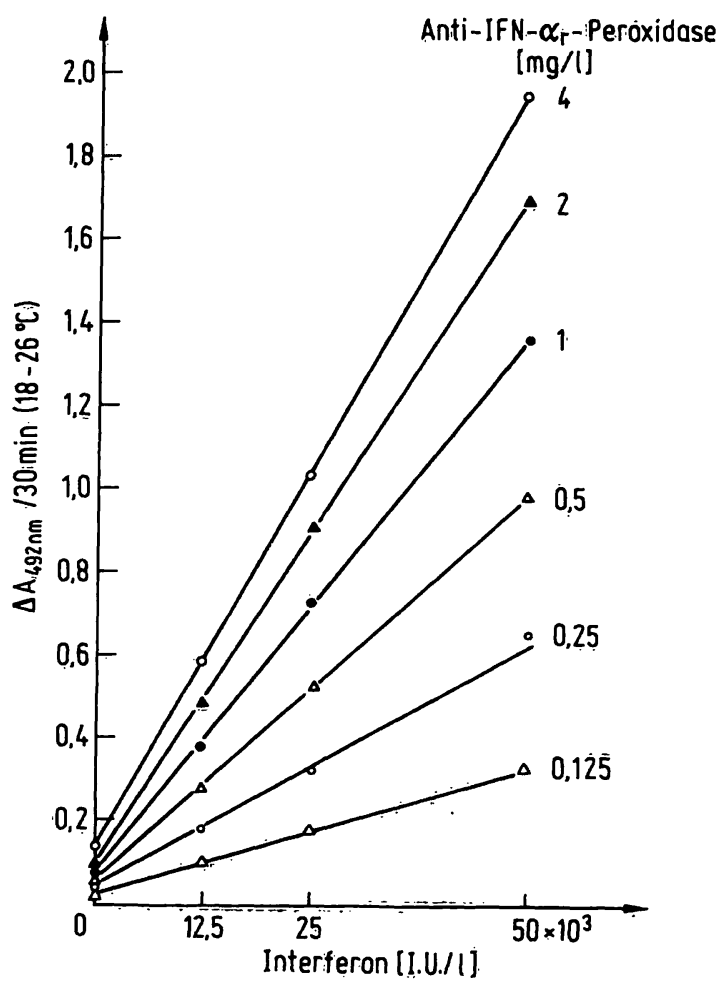

Abb. 3. Einfluß der Anti-Interferon=Peroxidasê-Konzentration auf den Intẹrfèrón-EIA.

Der Interféron-EIA wurde nach der allgemeinen Testvorschrift durchgeführt, außer daß̉ die Konżentràtionon an AntiInterferon-Peroxidase-Konjugat in der Testlösung entsprechend variiert wurde.

\section{Einfluß der Inkubationsdauer und der Inkubations- temperatur auf den Interferon-EIA}

Die Testempfindlichkeit des Interferon-EIA ist abhängig von der Dauer und der Temperatur der immunologischen Reaktion. Dabei ergibt sich überraschenderweise bei einer 16-stündigen Iñkubationsdauer die höchste Testempfindlichkeit bei einer Inkubationstemperatur von $2=8^{\circ} \mathrm{C}$. Bei $18-26^{\circ} \mathrm{C}$ ișt die Testempfindlichkeit etwas geringer, während sie bei einer Reäktions̄temperatur vo $37^{\circ} \mathrm{C}$ wesentlich abfällt (Abb. 4). Die unterschiedlichen Inkubationstemperaturen zeigen keinen Einfluß weder auf die Linearität der Interferon-Standardkurve noch auf den Reagenzienleerwert.

In Abbildung 5 ist die Kinetik der immunologischen Reaktion des Interferons mit den beiden Reaktionspartnern, dem an die Kugel adsorbierten Anti-HuIFN$\alpha_{\mathrm{i}}$ und dem Anti-HuIFN- $\alpha_{\mathrm{i}}$-Peroxidase-Konjugat, für die drei Inkubationstemperaturen $2-8^{\circ}{ }^{\circ} \mathrm{C}, 18=26^{\circ} \mathrm{C}$ 


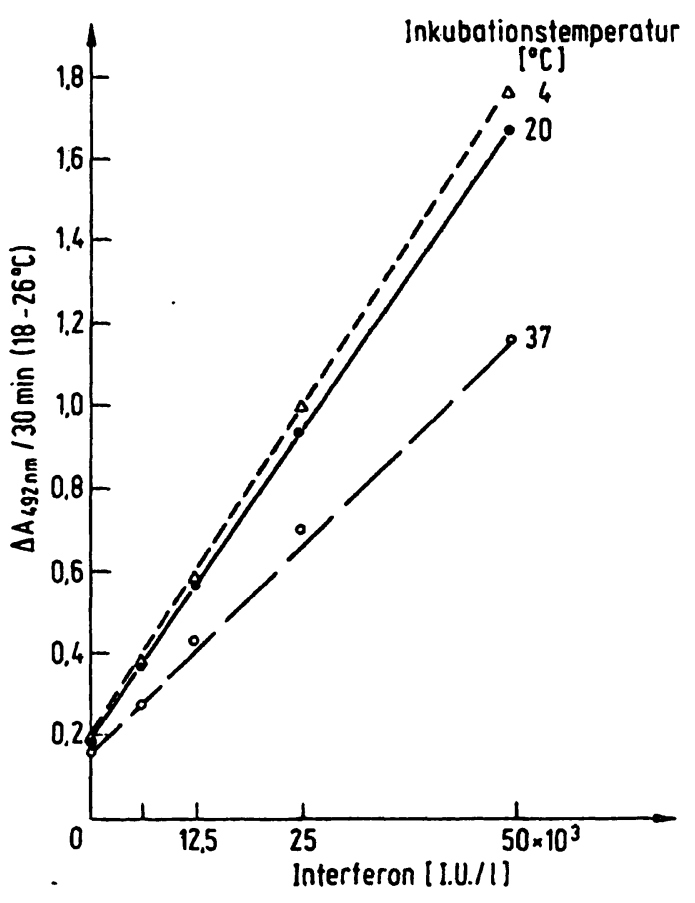

Abb. 4. Einfluß der Inkubationstemperatur der immunologischen Reaktion auf den Interferon-EIA.

Der Interferon-ElA wurde nach der allgemeinen Testvorschrift durchgefuhrt, außer daß die Inkubationstemperatur während der immunologischen Reaktion entsprechend variiert wurde. Die Inkubationsdauer betrug bei allen drei Versuchen 16 Stunden.

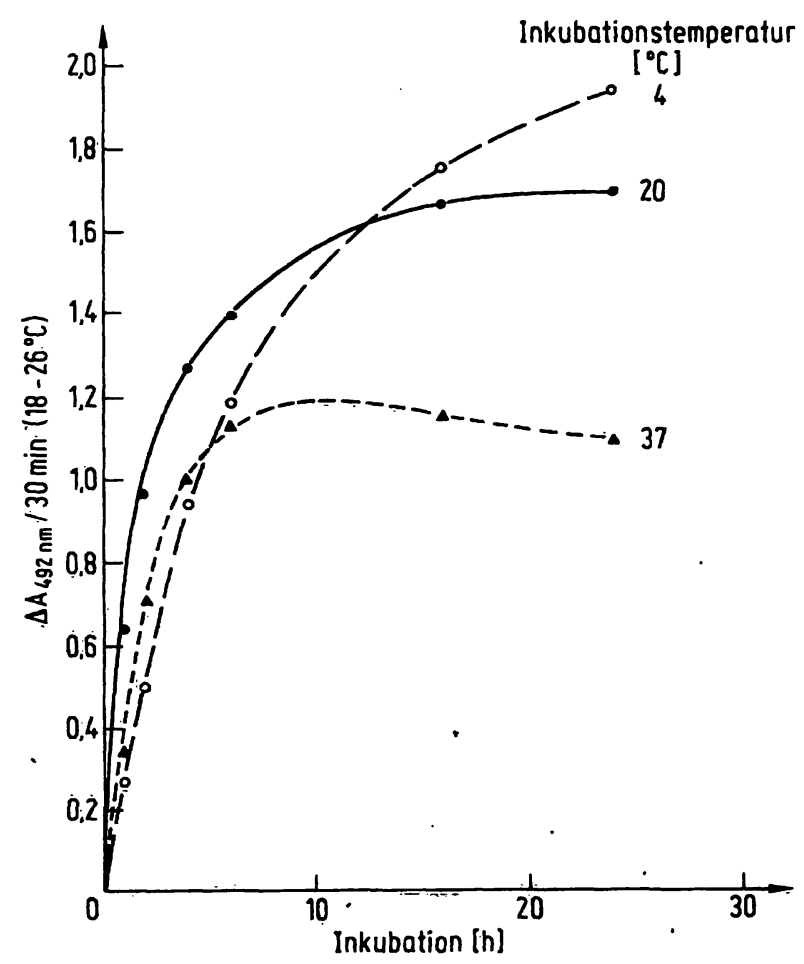

Abb. 5. Kinetik der immuñologischen Reaktion beim InterferonEIA in Abhängigkeit von der Inkubationstemperatur. Der Interferon-EIA wurde nach allgemeiner Testvorschrift durchgeführt, außer daß die Inkubationsdauer und die Inkubationstemperatur entsprechend variiert wurden. und $37^{\circ} \mathrm{C}$ dargestellt. Unter den gegebenen Reaktionsbedingungen scheint das Reaktionsgleichgewicht bei einer Inkubationstemperatur von $37^{\circ} \mathrm{C}$ schon nach 10 Stunden erreicht zu sein, während bei $2-8^{\circ} \mathrm{C}$ die immunologische Reaktion nach 24 Stunden noch nicht abgeschlossen ist. Bei dieser Reaktionskinetik ist besonders zu beachten, daß die maximal mögliche Testempfindlichkeit mit steigender Inkubationstemperatur abnimmt.

Die Kine tik der immunologischen Reaktion, wie sie in der Abbildung 5 dargestellt ist, macht die Relativität der Resultate der Abbildung 4 deutlich. Je nach der Inkubationsdauer werden - bezogen auf die drei untersuchten Inkubationstemperaturen - unterschiedliche Testempfindlichkeiten erreicht. Eine identische Testempfindlichkeit würde demnach für die Inkubationstemperaturen $2-8{ }^{\circ} \mathrm{C}$ und $18-26^{\circ} \mathrm{C}$ bei einer Inkubationsdauer von 12 Stunden erhalten.

Aus Gründen der Praktikabilität und der besseren Reproduzierbarkeit der Testresultate wird für die Durchführung des Interferon-EIA eine Inkubationstemperatur von $18-26^{\circ} \mathrm{C}$ (Raumtemperatur) sowie eine Inkubationsdauer von $16(10-24)$ Stunden vorgeschlagen.

\section{Testbedingungen der enzymatischen Indikatorreaktion}

Nach der immunologischen Reaktion wird das nicht gebundene Material durch einen Waschschritt abgetrennt, und die Kugeln werden in Peroxidasesubstrat-Puffer während 30 Minuten bei $18-26^{\circ} \mathrm{C}$ inkubiert. Entsprechend den optimierten Reaktionsbedingungen (23) enthält diese Substratpufferlösung $40 \mathrm{mmol} / 11,2$ Phenylendiamin und $6 \mathrm{mmol} / 1 \mathrm{H}_{2} \mathrm{O}_{2}$ in $0,1 \mathrm{~mol} / \mathrm{l} \mathrm{Kalium}$ citratpuffer vom $\mathrm{pH}$ 5,0. Durch die katalytische Reaktion der an die Kugel immunologisch gebundenen Peroxidase wird bei Anwesenheit von $\mathrm{H}_{2} \mathrm{O}_{2}$ das 1,2-Phenylendiamin zum gelb-braunen 2,2'-Diaminoazobenzol kondensiert.

Durch Zusatz von Salzsäure wird der pH-Wert des Peroxidasesubstrat-Puffers unter 1,0 gesenkt. Dadurch wird die Peroxidaseaktivität sogleich und vollständig abgestoppt und zudem die Farbintensität des gebildeten 2,2'-Diaminoazobenzols wesentlich erhöht.

\section{Enzym-immunologische Interferon-Bestimmung im Serum oder Plasma?}

Auf Grund der bisherigen Resultate sollte für die Bestimmung des Interferons nur Serum verwendet werden.

Interferon in Humanplasmen wird nur unvollständig und von Plasma zu Plasma sehr unterschiedlich erfaßt. Dagegen liegt die Wiederfindungsrate des Interferons in Humanseren bei $100 \%$ und es konnten innerhalb der bisherigen Studien keine individuellen Unterschiede von Serum zu Serum festgestellt werden. 
Eine partielle Hämolyse stört die Interferon-Bestimmung im Serum nicht.

Zur Stabilisierung von Interferon-Proben darf kein Natriumazid verwendet werden, da schon kleinste Mengen dieser Substanz die Peroxidase irreversibel inaktivieren.

Bestimmung des bakteriell gewonnenen und des aus Blut gereinigten Leukocyten-Interferons mit dem enzymimmunologischen Test

Das Interferon, das bei viralen Infektionen von den induzierten Leukocyten gebildet und sezerniert wird, ist nicht homogen. So konnten bis jetzt über 10 verschiedene Leukocyten-Interferone identifiziert werden. Es ist daher verständlich, daß die beiden im Interferon-EIA verwendeten monoklonalen Antikörper nicht mit allen natürlich vorkommenden Leukocyteninterferonen reagieren. Die Standardkurven der Abbildung 6 zeigen eine wesentlich größere immunologische Aktivität des Interferon-EIA mit dem bakteriell gewonnenen Leukocyteninterferon als mit dem aus Blut isolierten Leukocyten-Interferon. Die biologische, antivirale Aktivität der beiden untersuchten Leukocyten-Interferonlösungen (HuIFN- $\alpha$ und HuIFN- $\alpha_{\mathrm{r}}$ ) war identisch.

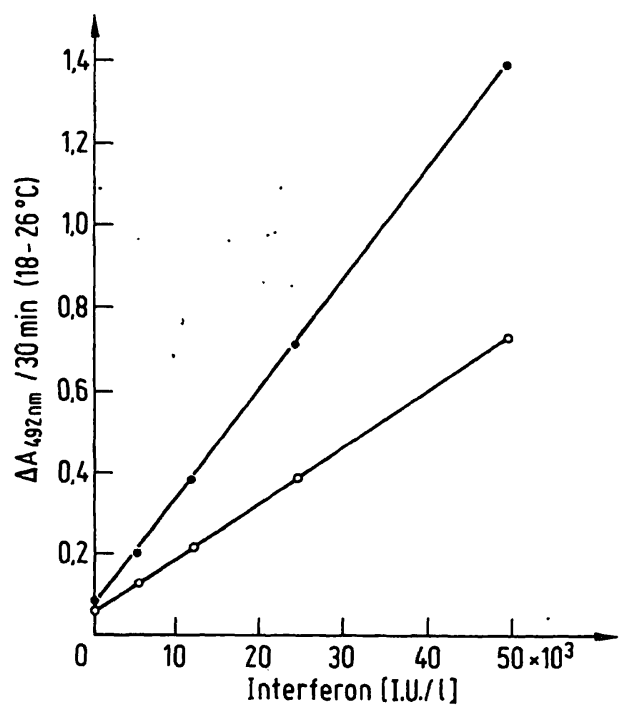

Abb. 6. Enzym-immunologische Bestimmung einer aus Blut isolierten Leukocyteninterferonprobe $(0-0)$ und einer bakteriell hergestellten Leukocyteninterferonlösung (•-•), beide Lösungen mit gleicher antiviraler Aktivität.

Der Interferon-EIA wurde nach allgemeiner Vorschrift einmal mit einer aus Blut isolierten Leukocyteninterferonprobe und einmal mit einer bakteriell hergestèlten Leukocyteninterferonlösung durchgefuihrt. Beide Lösungen wurden durch entsprechende Verdünnung auf die gleiche antivirale Aktivität gebracht.

\section{Téstempfindlichkeit des enzym-immunologischen Inter- ferontests}

Während der immunologischen Reaktion wird das Interferon aus der Probe an die mit Anti-HuIFN- $\alpha_{\mathrm{T}}$ beschichtete Kugel gebunden und mit Anti-HuIFN- $\alpha_{\mathrm{T}}$-Peroxidase-
Konjugat markiert. Wesentliche Voraussetzung für eine quantitative Erfassung des Interferons ist der intensive Kontakt der Testlösung mit der Kugeloberfläche.

Diese Voraussetzung wird erfüllt, wenn die InterferonBestimmung entsprechend der allgemeinen Testvorschrift mit einem Lösungsvolumen von $0,25 \mathrm{mil}^{\prime}(0,2 \mathrm{ml}$ Probe und 0,05 ml Testlösung) in Sarștedt-Röhrchen (75 X 11 $\mathrm{mm}$ ) durchgeführt wird.

Die oben erwähnte Voraussetziung wird aber auch dann erfüllt, wenn ein größeres Probenvolumen eingesetzt wird, dafür aber während der immunologischen Reaktion die Teströhrchen bewegt weṛden, so daß die beschichtete Kugel mit der gesamten Testlösung in Kontakt kommt. Durch die Vergrößerung des Probenvolumens kann die Empfindlichkeit des Interferon-EIA wesentlich gesteigert werden, so daß Werte bis 100 I.U./1 Interferon meßbar werden (Abb. 7).

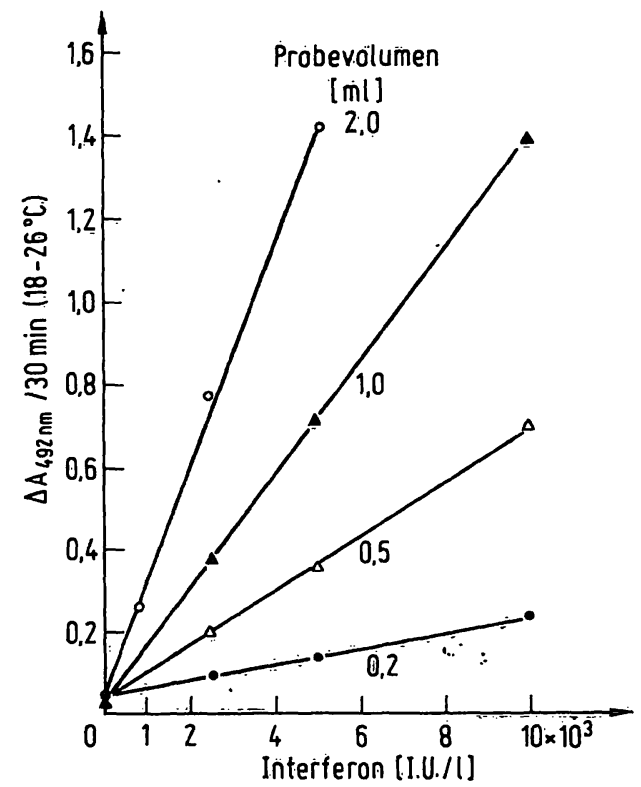

Abb. 7. Empfindlichkeit des Interferon-EIA in Abhängigkeit des eingesetzten Probevolumens.

Durchführung des Interferon-EIA nach allgemeiner Vorschrift, außer daß das Probevolumen entsprechend variiert und die Teströhrchen während der 16stündigen Inkubation leicht geschaukelt wurden.

Zur Einhaltung gleicher Testbedingungen muß mit dem Probenvolumen proportional auch das Volumen der Testlösung verändert werden. Andererseits kann der Meßbereich wesentlich erweitert und damit zusammenhängend die Tèstempfindlichkeit verringert werden; indem die Dauer der immunologischen Reaktion z.B. auf 30 Minuten verkürzt wird. Ein solcher ,Rapid-Test“ ist vor allem zur Uberwạchung der bakteriellen Produktion und der Reinigung von bakteriellem Interferon von praktischer Bedeutung. Die Abbildung 8 zeigt die Interferon-Standardkurve im Meßbereich von $0-10^{6}$ I.U:/1, wie sie mit dem „Rapid-Test“ erhalteñ wurde. Die Standardkurve ist nicht vollständig linear. 


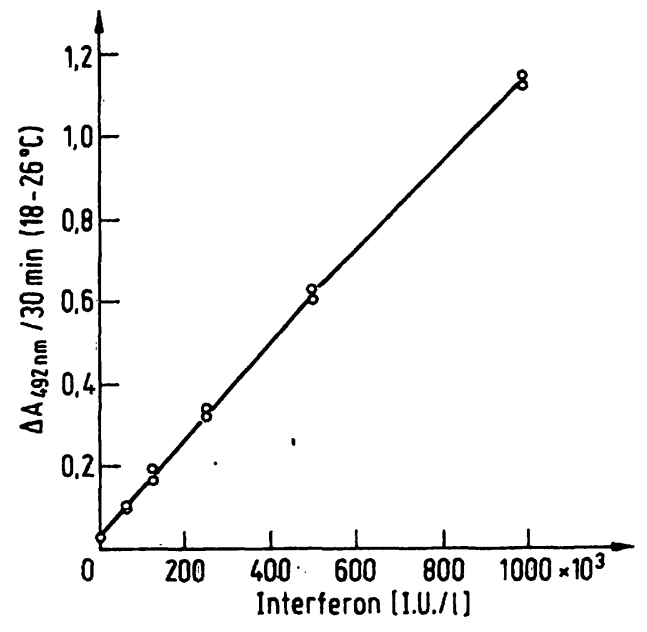

Abb. 8. Standardkurve des Interferon-EIA-,,Rapid“-Tests. Durchfuhrung des Interferon-EIA nach allgemeiner Vorschrift, außer daß die immunologische Reaktion auf 30 Minuten reduziert und der Meßbereich des Interferons auf $0-10^{6}$ I.U./1 ausgedehnt wurde.

\section{Reproduzierbarkeit des Interferon-EIA}

Die Ergebnisse der Reproduzierbarkeitsprüfung des Interferon-EIA innerhalb einer Serie von 20 Bestimmungen an Hand von drei unterschiedlich aufgestockten Humanseren sind in der Tabelle 1 zusammengefaßt. Die Variationskoeffizienten schwanken von $2,9 \%$ bis $6,1 \%$.

Tab. 1. Variationskoeffizient des Interferon-EIA.

Drei Seren mit unterschiedlichem Interferon-Gehalt wurden nach Vorschrift mit dem Interferon-EIA einer 20 fachen Bestimmung innerhalb einer Serie unterzogen.

\begin{tabular}{llll}
\hline & Serum & Serum & Serum \\
& $\mathrm{A}$ & $\mathrm{B}$ & $\mathrm{C}$ \\
\hline Interferon-Gehalt & $20 \times 10^{3}$ & $30 \times 10^{3}$ & $50 \times 10^{3}$ \\
& I.U./1 & I.U./1 & I.U./1 \\
Anzahl Bestimmungen & 20 & 20 & 20 \\
$\overline{\mathbf{x}}\left(\Delta \mathrm{A}_{492 \mathrm{~nm}} / 30 \mathrm{~min}\left(20^{\circ} \mathrm{C}\right)\right)$ & 0,897 & 1,244 & 2,078 \\
$\mathrm{~s}\left(\Delta \mathrm{A}_{492 \mathrm{~nm}} / 30 \mathrm{~min}\left(20^{\circ} \mathrm{C}\right)\right)$ & 0,054 & 0,063 & 0,060 \\
$\mathrm{VK}$ & $6,08 \%$ & $5,08 \%$ & $2,90 \%$ \\
\hline
\end{tabular}

\section{Diskussion}

Das Interferon kann therapeutische, diagnostische und vielleicht auch prophylaktische Bedeutung erlangen. Therapeutisch wird das Interferon zur Behandlung von viralen Infektionen und möglicherweise auch von speziellen Krebserkrankungen eingesetzt werden. Dazu sind allerdings größere Mengen von reinem Interferon notwendig, das heute in Bakterien ( $E$. coli) durch DNARekombinationstechnik hergestellt und affinitätschromatographisch gereinigt werden kann. Diagnostisch wird das Interferon zur Differenzierung von Infektionen interessant werden. Bei einem Virus-Befall ist die Interfèron-Konzentration im Blut (24-26) erhöht. Das Interferon könnte aber auch prophylaktische Bedeutung erlangen. Möglicherweise nimmt unter bestimmten Voraussetzungen die Fähigkeit zur Interferonbildung ab, so daß der Organismus den Virusinfektionen entsprechend schutzlos gegenüber steht. Durch geeignete Testanordnung könnte dieser Defekt diagnostiziert und durch entsprechende Interferongaben.korrigiert werden.

Für all diese Anwendungsgebiete des Interferons ist aber eine möglichst einfache, spezifissche und äußerst empfindliche Testmethode wesentliche Voraussetzung.

Der in dieser Arbeit vorgeschlagene enzym-immunologische Interferontest ist sehr einfach in der Durchführung. Bisher wurden die Festphasen-SandwichTests mit zwei separaten immunologischen Reaktionsschritten durchgeführt. In einem ersten Schritt reagierte das zu bestimmende Antigen mit einem entsprechenden Antikörper, der an eine feste Phase gebunden war. Nach einem Waschschritt wurde dann anschließend in einem zweiten Schritt das Antigen mit einem entsprechend markierten Antikörper zur Umsetzung gebracht. Inzwischen hat sich aber gezeigt, daß diese beiden Reaktionsschritte während einer einzigen Inkubation durchgefuihrt werden können. Dabei wird das Interferon gleichzeitig mit dem Peroxidase-markierten InterferonAntikörper und der mit Anti-Interferon beschichteten Polystyrolkugel inkubiert. Diese „Eintopf“-Testmethode ist prinzipiell immer dann möglich, wenn die zu bestimmende Substanz mindestens zwei verschiedene immunologische Bindungsstellen besitzt, wenn zwei Antikörper eingesetzt werden, die auf die beiden unterschiedlichen Epitope des Antigens ausgerichtet sind, und wenn die beiden Antikörper sich in ihrer Reaktion mit dem Antigen nicht gegenseitig behindern. Für diese „Eintopf “-Testmethode sind monoklonale Antikörper besonders geeignet.

Der enzym-immunologische Test bestimmt spezifisch jene Interferone, die von den vorhandenen Antikörpern erkannt werden. Beim hier beschriebenen Test werden monoklonale Antikörper eingesetzt. Daher ist es verständlich, daß mit diesem Testsystem nicht alle natürlich vorkommenden Leukocyteninterferone erfaßt werden. Prinzipiell kann aber durch die Wahl der Antikörper oder der Antikörpermischungen die gewünschte Spezifität des immunologischen Interferontests festgelegt werden. Bei der Interferon-Bestimmung ist die immunologische und die antivirale Aktivität zu unterscheiden. Während der immunologische Test molekularspezifisch ist, erfaßt der biölogische Interferontest jene Substanzklassen, die antivirale Aktivität besitzen.

Der Meßbereich und damit zusammenhängend die Testempfindlichkeit kann je nach den Anforderungen frei gewählt werden. So kann bei entsprechender Durchführung des Interferontests einerseits die Nachweisgrenze bis zu einer Konzentration von 100 I.U./1 Interferon gesenkt und andererseits der Meßbereich bis $10^{6}$ I.U./I Interferon ausgedehnt werden.

Die Reproduzierbarkeit dieses einfachen, enzymimmunologischen Interferontests ist erwartungsgemäß gut. Die Variationskoeffizienten innerhalb einer Serie betragen 2,9 bis $6,1 \%$. 


\section{Literatur}

1. Isaacs, A. \& Lindemann, J. (1957) Proc. R. Soc. London (Biol.) 147, 258-267.

2. Gresser, I. (1979) Interferon, Vol. I. London: Academic Press.

3. Priestman, T. (1979) Cancer Treatm. Rev. 6, 223-237.

4. Borecky, L. (1979) Acta Biol. Med. Germ. 38, 709-731.

5. Meurs, E. (1980) Bull. Institut.Pasteur 78, 175-212.

6. Krim, M. (1980) Blood 55, 711-721.

7. Krim, M. (1980) Blood 55, 875-884.

8. Stringfellow, D. (1980) Interferon and interferon inducers, New York: M. Dekker.

9. Pohl, A., Moser, K. \& Micksche, M. (1981) Wiener Klin. Wochenschrift 93, 439-457.

10. Goeddel, D., Yelverton, E., Ullrich, A., Heyneker, H., Miozzari, G., Holmes, W., Seeburg, P., Dull, T., May, L. Stebbing, N., Crea, R., Maeda, S., McCandliss, R., Sloma, A., Tabor, J., Gross, M., Familletti, P. \& Pestka, S. (1980) Nature 287, 411-416.

11. Taniguchi, T., Mantei, N., Schwarzstein, M., Nagata, S., Muramatsu, M. \&Weissmann, C. (1980) Nature 285, 547-549.

12. Nagata, S., Mantei, N. \& Weissmann, C. (1980) Nature $287,401-408$.

13. Goeddel, D., Leung, D., Dull, T., Gross, M., Lawn, R., McCandliss, R., Seeburg, P., Ullrich, A., Yelverton, E. \& Gray, P. (1981) Nature $290,20-26$.
14. Rubinstein, S., Familletti, P. \& Pestka, S. (1981) J. Virol. $37, \overline{7} 55-758$.

15. Armstrong, J. (1971) Appl. Microbiol: 21, 723-725.

16. Secher, D. \& Burke, D. (1980) Nature 285, 446-450.

17. Secher. D. (1981) Nature 290, 501-503,

18. Staehelin, Th., Durrer, B., Schmidt, J., Tacacs, B., Stocker, J., Miggiano, V., Stähli, C., Rubinstein, M., Levy, W., Hershberg, R. \& Pestka, S. (1981) Proc. Natl. Acad. Sci. USA 78, 1848-1852.

19. Voller, A., Bidwell, D. \& Bartlett, A. (1976) in Protides of the Biological Fluids, pp. 75i-758 (Peters, H., èd.) Pèrgamon Press, Oxford, U.K.

20. Kawoi, A. \& Nakane, P. (1972) Fed. Proc. 32, 840.

21. Nakane, P. (1975) Ann. N.Y. Acad. Sci. 254, 203-211.

22. Nakane, P. \& Kawoi, A. (1974) J. Histochem. Cytochem. $22,1084-1091$.

23. Gallati, H. \& Brodbeck, H. (1982) J. Clin. Chem. Clin. Biochem. 20, 221-225.

24. Matthews, T. \& Lawrence, M. (1979) Arch. Virol. 59, 35-38.

25. Schattner, A., Merlin, G., Levin, S., Wallach, D., Hahn, T. \& Revel, M. (1981) Lancet II, 497-499.

26. Parry, R. \& Parry, J. (1981) Lancet $I, 505-507$.

Dr. H. Gallati

Zèntrale Forschungseinheiten F. Hoffmann-La Roche \& Co. Grenząchersträße 124 CH-4002 Basel 